SLAC-PUB-6289

hep-ph/9310211

September 1993

$\mathrm{T} / \mathrm{E}$

\title{
Squark Mass Determination at the Next Generation of Linear $e^{+} e^{-}$Colliders 6
}

\author{
Jonathan L. Feng 4 : \\ Stanford Linear Accelerator Center \\ Stanford University, Stanford, California 94309
}

\begin{abstract}
Current mass limits allow the possibility that squarks may be produced in large numbers at the next generation of linear $e^{+} e^{-}$colliders. In this paper we investigate the prospects for precision studies of squark masses at such colliders. We assume that squarks are lighter than gluinos, and discuss both direct and cascade decay scenarios. By exploiting the clean environment and polarizable beams of linear $e^{+} e^{-}$colliders, we find that squark mass determinations at the level of a few $\mathrm{GeV}$ are possible in a large part of the parameter space.
\end{abstract}

(Submitted to Physical Review D)

\footnotetext{
*Work supported by the Department of Energy, contract DE-AC03-76SF00515.

${ }^{\dagger}$ Work supported in part by an NSF Graduate Research Fellowship.

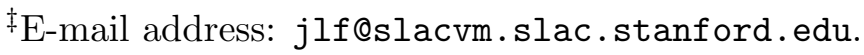

$\S$ E-mail address: dfinnell@slacvm.slac.stanford.edu.
} 


\section{INTRODUCTION}

Supersymmetry (SUSY) is at present a promising theoretical framework for physics beyond the Standard Model [1]. In addition to being free of quadratic divergences and providing a natural dark matter candidate, the simplest supersymmetric theory, the Minimal Supersymmetric Standard Model (MSSM), has had remarkable success in explaining the unification of coupling constants [2]. To solve the gauge hierarchy problem, SUSY must be broken at energies of order $1 \mathrm{TeV}$, and thus the supersymmetric particles of the MSSM must be within reach of the next generation of accelerators. This has stimulated a great deal of activity in MSSM phenomenology in recent years. It should be noted, however, that most of this activity has centered on particle searches. If supersymmetry is discovered, there will be a rich spectrum of superparticles, and detailed studies of their masses and couplings will be the focus of experimental particle physics into the next century. Precision measurements of such quantities are crucial to the understanding of the SUSY breaking sector of the MSSM and may even inform attempts to understand the mechanism of SUSY breaking in supergravity and string theories. It is not, then, premature to investigate the prospects for detailed study of superparticle properties at future facilities.

SUSY events are commonly characterized by unobservable particles in the final state, and it is therefore not obvious that the underlying SUSY parameters may be extracted from them with enough precision to be of theoretical interest. To date there has been relatively little work in this direction, with the exception of recent precision studies of sleptons and neutralinos/charginos [3, 4, 5, 6, 7]. These studies have shown that if such particles are pair-produced at a future $e^{+} e^{-}$collider, their masses could indeed be determined with enough accuracy to be significant tests of grand unified supergravity models. These particles are a natural starting point, as they are expected to be the lightest in the supersymmetric spectrum and therefore the most likely to be accessible at $e^{+} e^{-}$colliders with $\sqrt{s}=500 \mathrm{GeV}-1 \mathrm{TeV}$. It is also possible, however, that squarks may be light enough to be produced at such machines. This scenario is allowed by current experimental limits 8 and, if true, would provide an extremely fertile ground for future experiments. Present limits allow squarks with masses above $100 \mathrm{GeV}$. With the expected features of the next $e^{+} e^{-}$collider, luminosities of $10 \mathrm{fb}^{-1} /$ year and beam energies of $250 \mathrm{GeV}$ [3, 9, 10], these would be pair-produced at the rate of thousands per year. More massive squarks would have to wait for accelerator upgrades, but their study would be qualitatively similar. Although squark searches have been considered in great detail in the context of hadron colliders [11, 12, 13, 14, 15, 16, precision studies at these machines would be very difficult. Not only must one control large hadronic backgrounds, but one must also work in a situation where only a few features of a complex event are reconstructed. In contrast, the clean environment of $e^{+} e^{-}$accelerators makes them promising for such studies. An added advantage is the availability of polarized beams, which, as we will see, is a very useful tool. The purpose of this article is to survey the prospects for precise determination of squark masses in the $e^{+} e^{-}$environment.

The discussion is organized as follows: In Sec. II we review relevant information about squarks and the MSSM. We will note precisely which simplifying assumptions of the MSSM we use in our analysis. In Sec. III we describe our event simulation and organize our exploration of the SUSY parameter space. In Sec. IV we treat the simplest region, in which squarks decay directly to the lightest supersymmetric particle (LSP). The more complicated 
regions with cascade decays are investigated in Secs. $\mathrm{V}$ and $\mathrm{VI}$, where the light neutralinos are gaugino-like and higgsino-like, respectively. In Sec. VII we extend the analysis to the case of higher mass squarks and higher energy colliders. We conclude with some final remarks in Sec. VIII.

\section{SQUARKS IN THE MSSM}

The MSSM is the simplest extension of the Standard Model that incorporates supersymmetry. Here we concentrate on the salient features for squarks and their decays. Full discussions may be found in a number of articles [1, 17].

We will use hats to denote superfields and tildes to denote superpartners of Standard Model particles. The MSSM includes the usual matter superfields and two Higgs doublet superfields

$$
\hat{H}_{1}=\left(\begin{array}{c}
\hat{H}_{1}^{0} \\
\hat{H}_{1}^{-}
\end{array}\right) \quad \text { and } \quad \hat{H}_{2}=\left(\begin{array}{c}
\hat{H}_{2}^{+} \\
\hat{H}_{2}^{0}
\end{array}\right)
$$

where $\hat{H}_{1}$ and $\hat{H}_{2}$ give masses to the isospin $-\frac{1}{2}$ and $+\frac{1}{2}$ fields, respectively. These two superfields are coupled in the superpotential through the term $-\mu \epsilon_{i j} \hat{H}_{1}^{i} \hat{H}_{2}^{j}$, where $\mu$ is the supersymmetric Higgs mass parameter. Both $\mu$ and $\tan \beta \equiv\left\langle H_{2}^{0}\right\rangle /\left\langle H_{1}^{0}\right\rangle$ will be important for our analysis of squark decays. Soft supersymmetry breaking terms [18] for scalars and gauginos are included in the MSSM with

$$
V_{\text {soft }}=\sum_{i} M_{i}^{2}\left|\phi_{i}\right|^{2}+\frac{1}{2}\left\{\left[M_{1} \tilde{B} \tilde{B}+\sum_{j=1}^{3} M_{2} \tilde{W}^{j} \tilde{W}^{j}+\sum_{k=1}^{8} M_{3} \tilde{g}^{k} \tilde{g}^{k}\right]+\text { h.c. }\right\},
$$

where $i$ runs over all scalars.

For every flavor of quark in the Standard Model, the MSSM contains both a left- and a right-handed squark whose masses are given by

$$
\begin{aligned}
& m_{\tilde{\tilde{u}}_{L}}^{2}=M_{\tilde{\tilde{L}}}^{2}+m_{u}^{2}+m_{Z}^{2} \cos 2 \beta\left(\frac{1}{2}-\frac{2}{3} \sin ^{2} \theta_{W}\right) \\
& m_{\tilde{\tilde{u}}_{R}}=M_{\tilde{U}}^{2}+m_{u}^{2}+\frac{2}{3} m_{Z}^{2} \cos 2 \beta \sin ^{2} \theta_{W} \\
& m_{\tilde{d}_{L}}^{2}=M_{\tilde{Q}}^{2}+m_{d}^{2}-m_{Z}^{2} \cos 2 \beta\left(\frac{1}{2}-\frac{1}{3} \sin ^{2} \theta_{W}\right) \\
& m_{\tilde{d}_{R}}^{2}=M_{\tilde{D}}^{2}+m_{d}^{2}-\frac{1}{3} m_{Z}^{2} \cos 2 \beta \sin ^{2} \theta_{W},
\end{aligned}
$$

where we have suppressed generational indices and have neglected left-right mixing terms which are relevant only for the top and bottom squarks. The terms $M_{\tilde{Q}}, M_{\tilde{U}}$, and $M_{\tilde{D}}$ are some of the soft supersymmetry breaking terms $M_{i}$ of Eq. 2, and the remaining terms are model independent and known once $\tan \beta$ has been determined. Accurate determination of squark masses thus allows determination of the SUSY breaking parameters of the squark sector, which are of great theoretical interest.

In the most general form of the MSSM, the different SUSY breaking parameters are unrelated. To satisfy flavor changing neutral current constraints, however, corresponding terms in the first and second generations must be nearly degenerate [19]. (Recent work has noted that adequate squark degeneracy may be enforced by gauged horizontal symmetries [20] or 
may in fact be unnecessary to satisfy the FCNC constraints 21].) In minimal low energy supergravity models, the SUSY breaking parameters are usually related by assuming that they evolve from a universal scalar mass at a high scale. Renormalization group evolution then predicts a splitting between the left- and right-handed terms due to the contribution of $S U(2)$ gaugino loops, which can be of order $5 \%$ or higher depending on the gaugino mass, as well as a splitting of the third generation squarks from the first two. However, this assumption of a universal scalar mass is not on firm theoretical footing, and one of the goals of precision mass determinations is to differentiate between the universal scalar mass scenario and one where the different masses are not so simply related. We therefore do not assume a universal scalar mass.

The experimental signature of squark production will depend crucially on the details of the squark decay channels. As we will see below, the particles of the Higgs and gaugino sectors appear as intermediate decay states, and it is therefore necessary to discuss these sectors of the theory. The Higgs sector consists of two CP-even scalars $h^{0}$ and $H^{0}$, the charged scalar $H^{ \pm}$, the CP-odd scalar $A^{0}$, and the Higgsinos $\tilde{H}_{1}^{-}, \tilde{H}_{2}^{+}, \tilde{H}_{1}^{0}$, and $\tilde{H}_{2}^{0}$. The Higgsinos mix with gauginos to form mass eigenstates. The charged mass terms are $\left(\psi^{-}\right)^{T} \mathbf{M}_{\tilde{\chi}^{ \pm}} \psi^{+}+$h.c., where $\left(\psi^{ \pm}\right)^{T}=\left(-i \tilde{W}^{ \pm}, \tilde{H}^{ \pm}\right)$and

$$
\mathbf{M}_{\tilde{\chi}^{ \pm}}=\left(\begin{array}{cc}
M_{2} & \sqrt{2} M_{W} \sin \beta \\
\sqrt{2} M_{W} \cos \beta & \mu
\end{array}\right) .
$$

The chargino mass eigenstates are $\tilde{\chi}_{i}^{+}=\mathbf{V}_{i j} \psi_{j}^{+}$and $\tilde{\chi}_{i}^{-}=\mathbf{U}_{i j} \psi_{j}^{-}$, where the unitary matrices $\mathbf{U}$ and $\mathbf{V}$ are chosen to diagonalize $\mathbf{M}_{\tilde{\chi}^{ \pm}}$. Neutral mass terms may be written as $\frac{1}{2}\left(\psi^{0}\right)^{T} \mathbf{M}_{\tilde{\chi}^{0}} \psi^{0}+$ h.c., where $\left(\psi^{0}\right)^{T}=\left(-i \tilde{B},-i \tilde{W}^{3}, \tilde{H}_{1}^{0}, \tilde{H}_{2}^{0}\right)$ and

$$
\mathbf{M}_{\tilde{\chi}^{0}}=\left(\begin{array}{cccc}
M_{1} & 0 & -M_{Z} \cos \beta \sin \theta_{W} & M_{Z} \sin \beta \sin \theta_{W} \\
0 & M_{2} & M_{Z} \cos \beta \cos \theta_{W} & -M_{Z} \sin \beta \cos \theta_{W} \\
-M_{Z} \cos \beta \sin \theta_{W} & M_{Z} \cos \beta \cos \theta_{W} & 0 & -\mu \\
M_{Z} \sin \beta \sin \theta_{W} & -M_{Z} \sin \beta \cos \theta_{W} & -\mu & 0
\end{array}\right) .
$$

The neutralino mass eigenstates are $\tilde{\chi}_{i}^{0}=\mathbf{N}_{i j} \psi_{j}^{0}$, where $\mathbf{N}$ diagonalizes $\mathbf{M}_{\tilde{\chi}^{0}}$. The four neutralinos in increasing order of mass are $\tilde{\chi}_{1}^{0}, \tilde{\chi}_{2}^{0}, \tilde{\chi}_{3}^{0}$, and $\tilde{\chi}_{4}^{0}$, and the two charginos, similarly ordered, are $\tilde{\chi}_{1}^{ \pm}$and $\tilde{\chi}_{2}^{ \pm}$.

The MSSM as reviewed above contains many arbitrary constants, and usually some simplifications are made. In our version of the MSSM we will employ the following assumptions: (a) We assume R-parity conservation, a common assumption that imposes baryon and lepton number conservation. R-parity conservation implies that the LSP is stable and must be among the decay products of any supersymmetric particle.

(b) We take the LSP to be the lightest neutralino, $\tilde{\chi}_{1}^{0}$. The LSP must be neutral, and the other candidate, the sneutrino $\tilde{\nu}$, is heavily disfavored if one assumes that the LSP makes up galactic dark matter [22]. With this assumption, squarks can either decay directly into an LSP and a quark,

$$
\tilde{q} \rightarrow q \tilde{\chi}_{1}^{0}
$$

or indirectly to an LSP through a chain of neutralinos and charginos, e.g., 


$$
\tilde{q} \rightarrow q^{\prime} \tilde{\chi}_{1}^{+} \rightarrow q^{\prime} \tilde{\chi}_{1}^{0}(q \bar{q}, \nu \bar{l}) \quad \text { or } \quad \tilde{q} \rightarrow q \tilde{\chi}_{2}^{0} \rightarrow q \tilde{\chi}_{1}^{0}(q \bar{q}, l \bar{l}, \nu \bar{\nu})
$$

The latter decays are called cascade decays. Even more complicated cascades involving $\tilde{\chi}_{3}^{0}$, $\tilde{\chi}_{4}^{0}$, and $\tilde{\chi}_{2}^{ \pm}$are possible. The LSP interacts very weakly and disappears from the detector like a neutrino, so direct LSP decays leave a distinctive signature of acoplanar jets + missing $p_{T}\left(\not p_{T}\right)$. In the case of cascade decays, there may be additional jets and leptons.

(c) We assume $m_{\tilde{q}}<\left|M_{3}\right|$ so that squarks do not decay through gluinos. Without this assumption, the decay $\tilde{q} \rightarrow q \tilde{g}$ would be possible and would in fact dominate. Since the gluino is strongly interacting, it would be accompanied by additional hadronic radiation and a weakened $\not p_{T}$ spectrum. In such a case, a different analysis from ours will be needed.

(d) In this discussion, we assume that the neutralino/chargino sector has already been explored. We will therefore keep this sector simple by assuming the unification of gauge constants and gaugino masses at some higher scale. This implies that even at lower scales we have the one-loop condition [23]

$$
\frac{M_{2}}{g_{2}^{2}}=\frac{3}{5} \frac{M_{1}}{g^{\prime 2}}=\frac{M_{3}}{g_{3}^{2}}
$$

Eq. 8, coupled with assumption (c), will make our analysis invalid in some areas of the $\left(\mu, M_{2}\right)$ plane, which will be noted below.

Because direct decays of the squarks to the LSP are characterized by two acoplanar jets with large $\not p_{T}$, they should be easy to resolve experimentally. For example, cutting events with $\not p_{T}<35 \mathrm{GeV}$ and $\theta_{\text {acop }}>150^{\circ}$ will eliminate the bulk of Standard Model backgrounds [24] while eliminating only $20 \%-40 \%$ of the signal events. The primary backgrounds after these cuts are $W^{+} W^{-}$production, $e^{ \pm} \nu W^{\mp}$ via $\gamma W$ fusion, and $\nu \bar{\nu} Z$ via $W W$ fusion. $W$ pair production is a background to direct squark decays when one $W$ decays leptonically and the charged lepton is either mistakenly included in a jet or goes undetected. In the former case one can eliminate events whose missing momentum and energy are consistent with an undetected neutrino, and in the latter case one can eliminate events whose visible mass is consistent with the $W$ mass. All in all, we expect $W$ pair production to be a less troublesome background for squark studies than it is for chargino and slepton studies. A more serious background is $e^{ \pm} \nu W^{\mp}$. Before cuts, this cross-section is roughly an order of magnitude above the signal, and because the electron tends to be lost down the beam pipe and the neutrino tends to be produced with large $p_{T}$, the cuts are not particularly effective. However, this background can be removed by a cut on the two jet invariant mass. By eliminating events with invariant mass less than $100 \mathrm{GeV}$ the background is effectively removed while much of the signal is retained. Such a cut also reduces the $\nu \bar{\nu} Z$ background to negligible levels. One might also be able to take advantage of the fact that these backgrounds tend to produce jets in the same hemisphere, while squark pair jets are preferentially in opposite hemispheres.

Cascade decays result in a wide variety of signals, and are therefore generally more difficult to isolate. However, in the analysis of this paper, we will concentrate primarily on events with two jets and isolated leptons. Backgrounds to such events consist largely of events where an on-shell $W$ or $Z$ decays hadronically, and these will therefore also be removed by a cut on the two jet invariant mass. One important exception to this is $t \bar{t}$ events, which should be well-understood. This is a background when both top quarks decay to $b l \nu$. We can reduce this background by anti-tagging $b$ quarks, and in our discussion of 
cascade decays, we will discuss the effectiveness of such a cut. This cut excludes $b$ and $t$ squarks from our analysis: however, for reasons outlined in the following section, the third generation of squarks will most likely require a separate analysis anyway.

In addition to the Standard Model backgrounds discussed above, there may also be SUSY backgrounds to consider, such as chargino and neutralino production. Chargino pairs are a background to 2-jet cascade events when one chargino decays through a hadronic $W$ decay and the other through a leptonic $W$ decay. Neutralino production can result in backgrounds to both direct and cascade squark decays. However, in both chargino and neutralino 2-jet events, the two jets are produced by an on- or off-shell $W$ and $Z$ decay, so the two jet invariant mass should be less than or of order the $W$ and $Z$ masses. Thus, the invariant mass cuts that reduce the Standard Model backgrounds should also effectively reduce the SUSY backgrounds. In any case, it is not unreasonable to hope that once the Standard Model and MSSM backgrounds are well understood, efficient cuts can be devised to isolate the squark signal.

In the remainder of this paper we will apply only the $\not p_{T}$ and acoplanarity cuts given above, namely $\not p_{T}<35 \mathrm{GeV}$ for all events and $\theta_{\text {acop }}>150^{\circ}$ for events with only two jets. A cut requiring the 2 -jet invariant mass to be greater than $100 \mathrm{GeV}$ has only a small effect on the distributions we will plot, though it decreases the total signal by roughly a factor of 3 .

\section{EVENT SIMULATION AND ORGANIZATION OF PARAMETER SPACE}

For the purposes of this exploratory study, we use a simple parton level Monte Carlo to simulate squark production and decay. We then simulate hadronization and detector effects by smearing quark jets with a detector resolution of $\sigma_{E}^{\text {had }} / E=50 \% / \sqrt{E}(E$ in GeV). The cuts on $\not p_{T}$ and $\theta_{\text {acop }}$ discussed above are implemented to separate Standard Model backgrounds. Beamstrahlung and initial state radiation are not included, and we assume $100 \%$ electron beam polarization.

Systematic errors in this study are of two kinds. There are, of course, systematic errors arising from hadronization and detector effects. As our modeling of these effects is rather crude, a detailed study of these errors will not be attempted here. In addition, there are errors arising from the uncertainty in the determination of the neutralino and chargino masses which enter our analysis. The effects of these errors on our ability to determine squark masses will be included in our discussion of the case of direct decays.

We must fix the squark masses for the Monte-Carlo simulations. To simplify the analysis, we assume that all left-handed squarks from the first two generations are degenerate, as are the right-handed squarks, and concentrate on the left-right splitting. This assumption may be relaxed without major changes to the analysis, but will result in greater complications in fitting the data. The third generation of squarks will require a separate analysis, as the heavy top mass may not allow direct decays of the stop to the LSP, and in addition there will be an appreciable left-right mixing. In most situations, the third generation of squarks can be separated with $b$ anti-tagging, so we will consider only the first two generations from here on.

The present lower mass limit on squarks is $\sim 100 \mathrm{GeV}$ from the CDF experiment [8] when the important effects of cascade decays have been included [14]. These limits assume $M_{3}<$ $400 \mathrm{GeV}$ and disappear for higher $M_{3}$. Squarks will be pair-produced by $e^{+} e^{-} \rightarrow(\gamma, Z) \rightarrow$ 
$\tilde{q} \tilde{\tilde{q}}$. At $e^{+} e^{-}$colliders with $\sqrt{s}=500 \mathrm{GeV}$, the production rate becomes substantial not far below the kinematic limit of $m_{\tilde{q}}=250 \mathrm{GeV}$. For our studies, we will choose $m_{\tilde{q}_{L}}=220 \mathrm{GeV}$ and $m_{\tilde{q}_{R}}=210 \mathrm{GeV}$. These values are significantly above the present mass bounds, but are also low enough to give $\approx 2500$ events per year, assuming two degenerate squark generations and unpolarized beams at a luminosity of $10 \mathrm{fb}^{-1} /$ year. The results for $m_{\tilde{q}}=400 \mathrm{GeV}$ and $\sqrt{s}=1 \mathrm{TeV}$ are qualitatively similar; we will address this case in Sec. VII.

In Fig. 1, we plot the cross sections for pair production of $\tilde{q}_{L} \overline{\tilde{q}}_{L}$ and $\tilde{q}_{R} \overline{\tilde{q}}_{R}$ from $e^{+} e^{-}$ annihilation, assuming polarized $e^{-}$beams. Note the slow rise of the cross section near threshold, characteristic of scalar particle production, which precludes the use of cross section measurements alone for precision mass determinations. The polarization dependence of the cross section is an important feature. We see that $e_{L}^{-}$beams produce $\tilde{q}_{L} \overline{\tilde{q}}_{L}$ pairs $91 \%$ of the time, and $e_{R}^{-}$beams produce $\tilde{q}_{R} \overline{\tilde{q}}_{R}$ pairs $91 \%$ of the time. Beam polarization is therefore an extremely effective way of separating the left- and right-handed squarks.

Since squarks are expected to be among the heaviest superparticles, one cannot ignore the possibility of cascade decays. We should therefore consider a representative variety of values of the neutralino/chargino sector parameters rather than just the limiting cases. To do this, we must organize our survey of the parameter space. Given the constraint (8), all chargino and neutralino masses are given as functions of $\tan \beta, \mu$, and one of the gaugino masses, which we will take to be $M_{2}$. Thus, for a given value of $\tan \beta$, squark decays are determined by the parameters $\left(\mu, M_{2}\right)$, and our task reduces to exploring squark decays in this parameter plane.

The discussion will be limited to the part of the plane with $0 \leq M_{2}<1 \mathrm{TeV}$ and $|\mu|<1 \mathrm{TeV}$. The constraint $M_{2} \geq 0$ may be imposed without loss of generality. The upper bound on $M_{2}$ results from the fact that $M_{2}$ is a SUSY breaking parameter, and for SUSY to naturally explain the electroweak scale, $M_{2}$ cannot be too large. Although $\mu$ is not a SUSY breaking parameter, a large value for $\mu$ would re-introduce the fine-tuning problem. For most of this study, we will fix $\tan \beta=2$. The analysis for higher $\tan \beta$ is not much different and will be deferred to Sec. VIII.

With the parameters chosen above, we may now begin our study in earnest by dividing the $\left(\mu, M_{2}\right)$ plane into regions with similar squark decay channels. The decay patterns will, of course, also be influenced by the Higgs and slepton masses. Since these masses are unknown, however, the boundaries separating regions with different squark decays through Higgs and sleptons are not fixed. For this reason, we first divide the parameter space into regions ignoring the Higgs and slepton decays; we will then consider the effects of varying the Higgs and slepton masses within each region. The regions are shown in Fig. 2 for $m_{\tilde{q}}=220 \mathrm{GeV}$. We can ignore the hatched regions in the upper left and right corners, because there the squarks are lighter than $\tilde{\chi}_{1}^{0}$, which violates our assumptions. The crosshatched region along the $M_{2}=0$ and $\mu=0$ axes is experimentally ruled out by lower bounds on superparticle masses [25,26]. In region 4, the squarks can decay to four or more of the neutralinos/charginos. This results in very complicated cascade patterns. Though our methods may, in principle, be extended to this region, we will not consider it further in this paper. In what remains of the plane, the situation is greatly simplified, because the three neutralinos/charginos to which the squark can decay are always $\tilde{\chi}_{1}^{0}, \tilde{\chi}_{2}^{0}$, and $\tilde{\chi}_{1}^{ \pm}$. The only two-body decays kinematically allowed in region 1 are those directly to the LSP. In regions 2 and 3 , squarks may also decay to either $\tilde{\chi}_{2}^{0}$ or $\tilde{\chi}_{1}^{ \pm}$or both. In region $2, \tilde{\chi}_{1}^{0}, \tilde{\chi}_{2}^{0}$, 
and $\tilde{\chi}_{1}^{ \pm}$are all dominated by their gaugino components. This region is further subdivided into region $2 \mathrm{a}$, where $\tilde{\chi}_{2}^{0}$ and $\tilde{\chi}_{1}^{ \pm}$may decay to the LSP through on-shell $W$ and $Z$ bosons, and region $2 \mathrm{~b}$, where decays through on-shell $W$ and $Z$ decays are not possible. In region $3, \tilde{\chi}_{1}^{0}, \tilde{\chi}_{2}^{0}$, and $\tilde{\chi}_{1}^{ \pm}$are all Higgsino-like, and again only decays through off-shell $W \mathrm{~s}$ and $Z \mathrm{~s}$ are possible. Finally, each region has a mirror region in the $\mu>0$ part of the plane. These mirror regions usually have the same decay patterns as their $\mu<0$ counterparts, so where this is true, we will analyze only the $\mu<0$ regions. The only exception is region 3 , and we will note the different behavior for $\mu>0$ at the end of Sec. V1. In Fig. 2 the representative points in each region that we will consider in detail in the following sections are marked.

In what follows, it will often be helpful to keep in mind the following approximate relationships [27]. At $M_{Z}, M_{1} \approx \frac{1}{2} M_{2}$ and $M_{3} \approx \frac{10}{3} M_{2}$. For $|\mu|, M_{2} \gg M_{Z}$,

$$
\begin{array}{ll}
m_{\tilde{\chi}_{1}^{0}} \approx \min \left\{|\mu|, \frac{1}{2} M_{2}\right\}, & m_{\tilde{\chi}_{2}^{0}} \approx \min \left\{|\mu|, M_{2}\right\}, \\
m_{\tilde{\chi}_{3}^{0}} \approx \max \left\{|\mu|, \frac{1}{2} M_{2}\right\}, & m_{\tilde{\chi}_{4}^{0}} \approx \max \left\{|\mu|, M_{2}\right\}, \\
m_{\tilde{\chi}_{1}^{ \pm}} \approx \min \left\{|\mu|, M_{2}\right\}, & m_{\tilde{\chi}_{2}^{ \pm}} \approx \max \left\{|\mu|, M_{2}\right\} .
\end{array}
$$

Note that in this approximation, $\tilde{\chi}_{2}^{0}$ and $\tilde{\chi}_{1}^{ \pm}$are virtually degenerate throughout the plane. We will take advantage of this fact in our analysis of cascade decays in Sec. $\mathrm{V}$.

It should also be noted that our assumption that the squarks are lighter than gluinos, coupled with the unification assumption of Eq. 8, implies that our analysis is only valid for $M_{2} \gtrsim 67 \mathrm{GeV}$ (above the dotted line in Fig. 2). Also, if one makes the further assumption that one can compute the squark mass by applying the renormalization group equations with the desert hypothesis, one obtains

$$
m_{\tilde{q}}^{2} \approx m_{0}^{2}+7 m_{1 / 2}^{2}
$$

where $m_{0}$ and $m_{1 / 2}$ are the squark mass and the gaugino mass at the unification scale $M_{U}$, respectively. Since $m_{0}^{2}>0$ and $m_{1 / 2} \approx 0.8 M_{2}$, this leads to the constraint $M_{2} \lesssim 0.5 m_{\tilde{q}}$, which in the present case implies that $M_{2} \lesssim 110 \mathrm{GeV}$.

\section{DIRECT DECAYS}

In region 1 only direct decays of the squarks to the LSP are allowed. For the sake of concreteness, we will perform our Monte-Carlo simulations at the representative point $\left(\mu, M_{2}\right)=(-500 \mathrm{GeV}, 300 \mathrm{GeV})$. The quark jet from the decay of the scalar particle $\tilde{q} \rightarrow q \tilde{\chi}_{1}^{0}$ will have a flat energy distribution with endpoints

$$
E_{\max , \min }=\frac{E_{b}}{2}\left(1 \pm \sqrt{1-\frac{m_{\tilde{q}}^{2}}{E_{b}^{2}}}\right)\left(1-\frac{M_{\tilde{\chi}_{1}^{0}}^{2}}{m_{\tilde{q}}^{2}}\right)
$$

where $E_{b}$ is the beam energy, and we have neglected the quark mass. Thus, in theory, squark masses can simply be deduced from the distribution's endpoints. Unfortunately, the simple flat shape will be changed by cuts, and finite detector resolution and hadronization will smear the endpoints. Of the 1764 (975) squark pair events produced by the $e_{L}^{-}\left(e_{R}^{-}\right)$ beam, 1294 (683) survive the cuts. The energy distribution of the individual jets from the 
surviving events is given in Fig. 3, where detector resolution effects have been included. We see that the location of the endpoints is rather ambiguous. One way to extract the squark mass is to apply a binned likelihood fit to the jet energy distribution, with the logarithm of the likelihood given by

$$
\ln \mathcal{L}\left(m_{\tilde{q}_{L}}, m_{\tilde{q}_{R}}\right)=\sum_{i} A_{i}\left(m_{\tilde{q}_{L}}, m_{\tilde{q}_{R}}\right) \ln B_{i}-B_{i},
$$

where the sum is over all bins, $A_{i}\left(m_{\tilde{q}_{L}}, m_{\tilde{q}_{R}}\right)$ is the expected number of events in bin $i$ given hypothetical squark masses $m_{\tilde{q}_{L}}$ and $m_{\tilde{q}_{R}}$, and $B_{i}$ is the measured number of events in bin $i$. The actual values of $m_{\tilde{q}_{L}}$ and $m_{\tilde{q}_{R}}$ are determined by maximizing $\ln \mathcal{L}$, and the statistical error of the determination is given by the width of the $\ln \mathcal{L}$ peak. For simplicity we leave $m_{\tilde{q}_{R}}$ fixed at its actual value and calculate $\ln \mathcal{L}\left(m_{\tilde{q}_{L}}\right)$. In the $\ln \mathcal{L}$ calculations, we have approximated the theoretically expected number of events $A_{i}$ by Monte-Carlo simulations with a very large number of events (typically 50,000). We find that the $m_{\tilde{q}_{L}}$ determination has a statistical error of $0.9 \mathrm{GeV}$ at $95 \% \mathrm{CL}$. These calculations have been performed assuming that the LSP mass is known. The expected statistical errors on the masses of $\tilde{\chi}_{1}^{ \pm}$and $\tilde{\chi}_{1}^{0}$ from chargino studies are 3.2 and $2.0 \mathrm{GeV}$, respectively, while slepton studies should give the LSP mass to $1.0 \mathrm{GeV}$ [3]. A shift of $1.0 \mathrm{GeV}$ in the LSP mass causes a shift of about $0.7 \mathrm{GeV}$ in the central value of the likelihood fit.

In the single jet energy spectrum used above, correlations between the energies and directions of the two jets of a given event are ignored, since each point in the distribution represents only one jet. It is thus worth thinking about whether there is a more efficient way to use the information contained in the event sample. One possibility is to retain the jet correlation information by using a two-dimensional binning, but this necessitates a large jump in computing time to obtain the $A_{i}$ distribution accurately.

Instead, the method we will use extensively is the following: For each event, we calculate the quantity $m_{\tilde{q}}^{\min }$, the minimum squark mass kinematically possible, given the two observed quark jet momenta. For each event, we then get one value of $m_{\tilde{q}}^{\min }$, and we apply a binned likelihood method to the $m_{\tilde{q}}^{\min }$ distribution. The quantity $m_{\tilde{q}}^{\min }$ is easily determined (see Fig. (1). We label the particle momenta as $\tilde{q}\left(p_{1}\right) \rightarrow \tilde{\chi}_{1}^{0}\left(p_{3}\right) q\left(p_{4}\right)$ and $\tilde{q}\left(p_{2}\right) \rightarrow \tilde{\chi}_{1}^{0}\left(p_{5}\right) q\left(p_{6}\right)$. The total visible momentum is then $p_{V}=p_{4}+p_{6}$. Because we know $E_{b}$ and have measured $E_{1}$ and $E_{2}$, we can determine the LSP energies $E_{3}$ and $E_{5}$. However, we also know the LSP mass, so we can find the magnitudes $\left|\vec{p}_{3}\right|$ and $\left|\vec{p}_{5}\right|$, and since $p_{3}+p_{5}=-p_{V}$, the vectors $\vec{p}_{3}$ and $\vec{p}_{5}$ are constrained to lie on a circle $C$. We then can calculate the angles $\gamma$ and $\delta$ shown in Fig. 1 . The minimum squark mass corresponds to the maximum possible $\left|\vec{p}_{2}\right|$, and is given by

$$
\left(m_{\tilde{q}}^{\min }\right)^{2}=E_{b}^{2}-\left|\vec{p}_{3}\right|^{2}-\left|\vec{p}_{4}\right|^{2}+2\left|\vec{p}_{3}\right|\left|\vec{p}_{4}\right|(\cos \gamma \cos \delta-\sin \gamma \sin \delta) .
$$

The distributions of $m_{\tilde{q}}^{\text {min }}$ for left- and right-polarized electron beams are sharply peaked at the underlying masses $m_{\tilde{q}_{L}}$ and $m_{\tilde{q}_{R}}$, respectively (see Fig. 5). As before, for simplicity we leave $m_{\tilde{q}_{R}}$ fixed at its actual value and calculate $\ln \mathcal{L}\left(m_{\tilde{q}_{L}}\right)$. We find that the statistical error of the $m_{\tilde{q}_{L}}$ determination is reduced to $0.4 \mathrm{GeV}$ at $95 \% \mathrm{CL}$. Thus, we see that the $m_{\tilde{q}}^{\min }$ method improves our statistical error significantly. If the cut $m_{2-\text { jet }}>100 \mathrm{GeV}$ is necessary to reduce background, our event sample is reduced by a factor of 3, but the statistical error increases only slightly to $0.5 \mathrm{GeV}$ at $95 \% \mathrm{CL}$. We note, however, that a shift of $1 \mathrm{GeV}$ in 
the LSP mass now shifts the central value of the likelihood fit by approximately $1.5 \mathrm{GeV}$. The $m_{\tilde{q}}^{\min }$ calculation is more strongly dependent on the LSP mass, and therefore places a higher premium on its accurate determination.

The greater power of the $m_{\tilde{q}}^{\min }$ distribution is manifested in its sharp peak, because even slight variations in $m_{\tilde{q}_{L}}$ and $m_{\tilde{q}_{R}}$ move these peaks enough to create large differences between $A_{i}$ and $B_{i}$ in some bins. This implies that $\ln \mathcal{L}$ falls rapidly from its maximum. There are a number of reasons for the sharp peak of the $m_{\tilde{q}}^{\min }$ distribution. Here we just note that, roughly speaking, momentum vectors lying on large circles $C$ may give mass minima both close and far from the actual squark mass, depending on where the momentum vectors lie on $C$. However, small circles give only accurate solutions, and thus the calculated minimum masses preferentially lie close to the actual underlying squark mass.

One potentially powerful feature of squark mass studies in this region is that, since both left- and right-handed squarks have identical decay channels, a direct comparison can be made to determine left-right mass splittings. The left- and right-handed squarks can be isolated using polarized beams, and systematic errors, which should effect both polarizations equally, should largely cancel in the ratio of their masses. One can therefore determine leftright mass splittings to greater accuracy than one can determine the actual values of the masses. This is in contrast to the case of slepton studies, where use of the left-polarized beam is hampered by a large $W^{+} W^{-}$background [4:7]. As was explained above in Sec. [1], we do not expect $W^{+} W^{-}$backgrounds to be a signifigant problem for squarks.

While it may come as no surprise that precise mass determinations can be made in the simple case of direct decays, the large squark masses expected make it likely that more complicated decays will be present. For a generic point in parameter space, with a large number of possible decay chains present simultaneously, it is important to determine whether it is still be possible to extract accurate squark masses from the more complicated signal. We now turn our attention to this question in representive regions of parameter space.

\section{GAUGINO-LIKE CASCADES}

In region 2 squarks have new decay channels through on-shell $\tilde{\chi}_{2}^{0}$ and $\tilde{\chi}_{1}^{ \pm}$. In this region, however, $\tilde{\chi}_{1}^{0} \approx \tilde{B}, \tilde{\chi}_{2}^{0} \approx \tilde{W}^{3}$, and $\tilde{\chi}_{1}^{ \pm} \approx \tilde{W}^{ \pm}$, so since right-handed squarks do not couple to $S U(2)$ gauginos, they still decay predominantly directly to the LSP. This may be seen from the branching ratios of $\tilde{u}_{L}$ and $\tilde{u}_{R}$, given in the contour plots of Fig. 6. Similar plots for down-type squarks differ little. The analysis for right-handed squarks is therefore simple. First we use the polarized $e_{R}^{-}$beam to isolate right-handed squarks. Contamination from left-handed squarks will be of order $10 \%$, and most of these will go through complicated decay channels and can be easily separated by considering only two-jet events. We then apply the analysis of region 1 with little degradation of statistics. As shown in Fig. 6, lefthanded squarks, unlike right-handed squarks, do decay predominantly through cascades in some parts of region 2. When this is the case, a separate analysis for left-handed squarks is necessary. The rest of this section will be concerned with left-handed squarks only.

As suggested at the end of Sec. III, we can use the near-degeneracy of $\tilde{\chi}_{2}^{0}$ and $\tilde{\chi}_{1}^{ \pm}$to simplify matters. Throughout region 2 , these two particles are typically degenerate to a few $\mathrm{GeV}$, and since 


$$
\left|m_{\tilde{\chi}_{2}^{0}}-m_{\tilde{\chi}_{1}^{ \pm}}\right| \ll \frac{1}{2} M_{2} \approx m_{\tilde{\chi}_{2}^{0}}-m_{\tilde{\chi}_{1}^{0}}
$$

phase space suppression allows us to safely ignore decays of $\tilde{\chi}_{2}^{0}$ to $\tilde{\chi}_{1}^{ \pm}$and vice versa. We are then left with two-step decays with squarks decaying to $\tilde{\chi}_{2}^{0}$ and $\tilde{\chi}_{1}^{ \pm}$, which then decay to the LSP through three-body modes mediated by $W$ or $Z$ bosons, sleptons, sneutrinos, squarks, or Higgs bosons. The resulting quark jet energy distribution is much more complicated than it was in the direct case, with quarks produced both at the primary vertices, i.e., the initial squark decay vertices, and in the later cascade decays.

However, by choosing appropriate cuts, we can reduce the problem to the case of direct decays. Recall that in the $m_{\tilde{q}}^{\min }$ analysis of region 1 , we needed to know only the energies and momenta of the quarks leaving the primary vertices and the mass of the neutralino or chargino leaving those vertices, which we now denote $m_{\tilde{\chi}}^{\text {primary }}$. In the case of region 1 , such identifications are obvious, since in all events both of the quark jets are produced at the primary (and only) vertices, and $m_{\tilde{\chi}}^{\text {primary }}=m_{\tilde{\chi}_{1}^{0}}$ is always the correct choice. Cascade decays complicate this analysis, because the initial squark decay may involve neutralinos/charginos other than the LSP, and there may be many quarks produced, making it difficult to determine which two came from the primary vertices. Our stategy will be to find cuts that allow us to accurately identify the primary quarks and assign $m_{\tilde{\chi}}^{\text {primary }}$. We can then calculate $m_{\tilde{q}}^{\text {min }}$ for each event and proceed as in the previous section. Two simple strategies are (1) separating direct and cascade decays kinematically and choosing events that contain direct decays on both sides, and (2) considering double cascade events in which the $\tilde{\chi}_{2}^{0}$ or $\tilde{\chi}_{1}^{ \pm}$has only leptonic decay products. We will show that these two strategies are always sufficiently effective. We now consider regions $2 \mathrm{a}$ and $2 \mathrm{~b}$ in turn.

Decays of $\tilde{\chi}_{2}^{0}$ and $\tilde{\chi}_{1}^{ \pm}$through on-shell $W$ s and $Z_{\mathrm{s}}$ are allowed in region $2 \mathrm{a}$. We will take the representative point to be $\left(\mu, M_{2}\right)=(-500 \mathrm{GeV}, 200 \mathrm{GeV})$. At this point, the masses of $\tilde{\chi}_{1}^{0}, \tilde{\chi}_{2}^{0}$, and $\tilde{\chi}_{1}^{ \pm}$are $103.2,206.1$, and $206.0 \mathrm{GeV}$, respectively. The jet energy distribution is shown in Fig. 7, where every quark jet produced in the Monte-Carlo squark decays is binned separately. The distribution consists of three parts - a large peak of soft quarks emanating from squarks decaying to $\tilde{\chi}_{2}^{0}$ and $\tilde{\chi}_{1}^{ \pm}$(long dashes), a flat distribution from direct LSP decays (solid), and a wide hump from hadronic $W$ and $Z$ decays (short dashes). The total distribution is given by the dotted histogram. The soft jets in the peak may not be discernible experimentally, but our analysis will use only jets from direct decays, and these jets have energies that are always greater that $40 \mathrm{GeV}$.

As noted previously, since region 2 allows cascade decays, top quark production is potentially a troublesome background. In Fig. 8, we again plot the total jet energy distribution resulting from squark decays, but this time, for comparison, also the bottom quark spectrum resulting from top decays. In the bottom quark spectrum, we have removed all $b$ quarks produced in top events in which at least one $b$ quark has been successfully tagged. We assume $m_{\text {top }}=150 \mathrm{GeV}$ and a $b$-tagging efficiency of $80 \%$ [3]. The top quark background is substantially reduced below the signal, and as top quark decays will be well-understood by the time of these squark studies, the top quark background should not be a significant obstacle.

The branching ratios at $\left(\mu, M_{2}\right)=(-500 \mathrm{GeV}, 200 \mathrm{GeV})$ are 


$$
\begin{array}{ll}
\operatorname{BR}\left(\tilde{u}_{L} \rightarrow u \tilde{\chi}_{1}^{0}\right)=58 \%, & \operatorname{BR}\left(\tilde{d}_{L} \rightarrow d \tilde{\chi}_{1}^{0}\right)=36 \% \\
\operatorname{BR}\left(\tilde{u}_{L} \rightarrow u \tilde{\chi}_{2}^{0}\right)=28 \%, & \operatorname{BR}\left(\tilde{d}_{L} \rightarrow d \tilde{\chi}_{2}^{0}\right)=43 \% \\
\operatorname{BR}\left(\tilde{u}_{L} \rightarrow d \tilde{\chi}_{1}^{+}\right)=14 \%, & \operatorname{BR}\left(\tilde{d}_{L} \rightarrow u \tilde{\chi}_{1}^{-}\right)=21 \%
\end{array}
$$

so we see that there are still many direct LSP decays. We will therefore try to isolate the double direct LSP decay events and then apply the analysis of Sec. IV. Of the 1764 events produced with a left-polarized beam in $1 / 2$ year, 1437 pass the $\not p_{T}$ and $\theta_{\text {acop }}$ cuts. We then consider only events with 2 jets and no isolated leptons, leaving 673 events, and for each of these we calculate $m_{\tilde{q}}^{\min }$ using $m_{\tilde{\chi}}^{\text {primary }}=m_{\tilde{\chi}_{1}^{0}}$. Of the remaining events, 244 are actually cascade events with neutrino decay products, but these may be removed by considering only events in which both jets have energy above $30 \mathrm{GeV}$. We find that the likelihood fit to the remaining $m_{\tilde{q}}^{\min }$ distribution gives squark masses to $1.3 \mathrm{GeV}$ at $95 \%$ CL.

The distribution of Fig. 7 was calculated for the case where the sleptons and Higgs scalars are massive enough that their diagrams are off-shell and suppressed. Smaller slepton and Higgs masses will, of course, change the part of the jet distribution resulting from cascades, but will have no effect on the two-jet event sample and will therefore not change our results.

We now turn to region $2 \mathrm{~b}$, whose representative point we take to be $\left(\mu, M_{2}\right)=$ $(-500 \mathrm{GeV}, 100 \mathrm{GeV})$. Here the masses of $\tilde{\chi}_{1}^{0}, \tilde{\chi}_{2}^{0}$, and $\tilde{\chi}_{1}^{ \pm}$are $52.8,108.1$, and $107.8 \mathrm{GeV}$, respectively, and we see that the $W$ and $Z$ diagrams are indeed off-shell. The branching ratios are

$$
\begin{array}{ll}
\operatorname{BR}\left(\tilde{u}_{L} \rightarrow u \tilde{\chi}_{1}^{0}\right)=7 \%, & \operatorname{BR}\left(\tilde{d}_{L} \rightarrow d \tilde{\chi}_{1}^{0}\right)=1 \%, \\
\operatorname{BR}\left(\tilde{u}_{L} \rightarrow u \tilde{\chi}_{2}^{0}\right)=61 \%, & \operatorname{BR}\left(\tilde{d}_{L} \rightarrow d \tilde{\chi}_{2}^{0}\right)=67 \%, \\
\operatorname{BR}\left(\tilde{u}_{L} \rightarrow d \tilde{\chi}_{1}^{+}\right)=32 \%, & \operatorname{BR}\left(\tilde{d}_{L} \rightarrow u \tilde{\chi}_{1}^{-}\right)=32 \% .
\end{array}
$$

Because there are not many direct LSP decays, the analysis must rely on cascade events. This complicates the analysis. However, we now show that even for the most difficult sets of parameters, a significant number of $\tilde{\chi}_{2}^{0}$ and $\tilde{\chi}_{1}^{ \pm}$decays will be purely leptonic. In these events we may unambiguously identify the two primary vertex quark jets, and we can then again apply the region 1 analysis.

To do this we must analyze the relative importance of the various cascade diagrams (see Fig. 9). The Higgs diagrams may be safely ignored. Typical values for the mass of the lightest Higgs scalar, $h^{0}$, are in the range $70-110 \mathrm{GeV}$. For this entire range, the $h^{0}$ process proceeds off-shell and is also suppressed by the bottom quark Yukawa coupling. The other Higgs scalars are significantly more massive and can also be safely ignored. In addition, we have squark and slepton cascade diagrams. Sleptons are generally expected to be lighter than squarks, and we will see below that the lower the slepton masses, the simpler our analysis. For now, we will pessimistically take the sleptons to be degenerate and of mass $200 \mathrm{GeV}$.

Since we have squark and slepton masses significantly higher than $M_{W}$, one might expect the $W$ and $Z$ diagrams to dominate. However, in the gaugino-like region of parameter space, $\left|\mathbf{N}_{11}\right| \approx\left|\mathbf{V}_{11}\right| \approx 1$, so the LSP is primarily composed of $\tilde{B}$, which does not couple to the $W$ or $Z$ at all. Thus, these diagrams are suppressed by a factor $S \equiv \mathcal{O}\left(\left|\mathbf{V}_{12}\right|,\left|\mathbf{N}_{12}\right|,\left|\mathbf{N}_{13}\right|,\left|\mathbf{N}_{14}\right|\right)$. We can obtain a rough estimate of $S$ by taking an appropriate limit of the explicit expression for $\mathbf{V}_{12}$ [27]. Taking $M_{W}$ small compared to $\mu$ and $M_{2}$, we have

$$
\mathbf{V}_{12} \approx \sqrt{2} M_{W} \frac{\mu \cos \beta+M_{2} \sin \beta}{M_{2}^{2}-\mu^{2}} \approx-\frac{50 \mathrm{GeV}}{\mu}\left(1+\frac{2 M_{2}}{\mu}\right),
$$


for $\tan \beta=2$, where we have expanded in terms of $M_{2} / \mu$, a small parameter in the gauginolike region. Thus, $\mathbf{V}_{12}$ is about 0.02 at our representative point. The $\mathbf{N}_{1 j}$ are roughly of the same order, and we find that $0.01 \lesssim S \lesssim 0.1$ throughout most of the region. The competing suppressions of the different diagrams make it impossible to simply determine which diagram dominates. In fact, our calculations show the gauge boson, squark, and slepton diagrams to be roughly of the same order. (Note that if one takes the gaugino-like relations $\tilde{\chi}_{1}^{0} \approx-i \tilde{B}$, $\tilde{\chi}_{2}^{0} \approx-i \tilde{W}^{3}$, and $\tilde{\chi}_{1}^{ \pm} \approx-i \tilde{W}^{ \pm}$too seriously, one is led to incorrectly conclude that the $W$ and $Z$ diagrams may be set to zero. This is never valid in the part of the plane we are considering.)

Evaluating the various diagrams with $m_{\tilde{l}}=m_{\tilde{\nu}}=200 \mathrm{GeV}$, we find

$$
\begin{array}{ll}
\operatorname{BR}\left(\tilde{\chi}_{2}^{0} \rightarrow q \bar{q} \tilde{\chi}_{1}^{0}\right)=20 \%, & \operatorname{BR}\left(\tilde{\chi}_{1}^{ \pm} \rightarrow q^{\prime} \bar{q} \tilde{\chi}_{1}^{0}\right)=55 \% \\
\operatorname{BR}\left(\tilde{\chi}_{2}^{0} \rightarrow l \bar{l} \tilde{\chi}_{1}^{0}\right)=31 \%, & \operatorname{BR}\left(\tilde{\chi}_{1}^{ \pm} \rightarrow l \nu \tilde{\chi}_{1}^{0}\right)=45 \% \\
\operatorname{BR}\left(\tilde{\chi}_{2}^{0} \rightarrow \nu \bar{\nu} \tilde{\chi}_{1}^{0}\right)=49 \% &
\end{array}
$$

We see that there are many leptonic decays, and we can therefore consider only the events with two quark jets + leptons without a great loss in statistics. (Note that at this point in the parameter plane the only jets below $40 \mathrm{GeV}$ are those produced by $W \mathrm{~s}$ and $Z \mathrm{~s}$. Thus by selecting only two-jet events, the jets that enter our analysis are again all hard enough to be experimentally detected.) Of the $1508 e_{L}^{-}$polarized events that pass the $\not p_{T}$ and $\theta_{\text {acop }}$ cuts, 834 have exactly 2 quark jets (+ leptons). Because the polarized beam has eliminated most right-handed squarks from our sample, and left-handed squarks rarely decay directly to the LSP, we expect that most of these events are double cascade events, and in fact

$70 \%$ are. We then calculate $m_{\tilde{q}}^{\min }$ with the assignment $m_{\tilde{\chi}}^{\text {primary }}=m_{\tilde{\chi}_{1}^{ \pm}}$. Note that since $\tilde{\chi}_{2}^{0}$ and $\tilde{\chi}_{1}^{ \pm}$are virtually degenerate, we need not distinguish them for the kinematic analysis of Sec. IV. About half of the events with one or two direct LSP decays are kinematically incompatible with the assignment $m_{\tilde{\chi}}^{\text {primary }}=m_{\tilde{\chi}_{1}^{ \pm}}$, and we eliminate these. Almost none of the double cascade events are removed by this cut. We are then left with 689 events, of which $84 \%$ are double cascade events. The double cascade events form a sharp peak in the $m_{\tilde{q}}^{\min }$ distribution, while the few events involving direct decays are much more broadly distributed. After performing a likelihood fit to the $m_{\tilde{q}}^{\min }$ distribution, we find that the squark mass can be determined to $2.4 \mathrm{GeV}$ at $95 \% \mathrm{CL}$.

If we lower the slepton mass, the slepton diagram contribution grows. For $m_{\tilde{l}}=110 \mathrm{GeV}$, even though the sleptons are still off-shell, the gauge boson suppression factor $S$ defined earlier allows the slepton diagrams to dominate, and the resulting branching ratios are

$$
\begin{array}{ll}
\operatorname{BR}\left(\tilde{\chi}_{2}^{0} \rightarrow q \bar{q} \tilde{\chi}_{1}^{0}\right)=0.3 \%, & \operatorname{BR}\left(\tilde{\chi}_{1}^{ \pm} \rightarrow q^{\prime} \bar{q} \tilde{\chi}_{1}^{0}\right)=6 \%, \\
\operatorname{BR}\left(\tilde{\chi}_{2}^{0} \rightarrow l \bar{l} \tilde{\chi}_{1}^{0}\right)=39.8 \%, & \operatorname{BR}\left(\tilde{\chi}_{1}^{ \pm} \rightarrow l \nu \tilde{\chi}_{1}^{0}\right)=94 \%, \\
\operatorname{BR}\left(\tilde{\chi}_{2}^{0} \rightarrow \nu \bar{\nu} \tilde{\chi}_{1}^{0}\right)=59.9 \% . &
\end{array}
$$

Clearly most $\tilde{\chi}_{2}^{0}$ and $\tilde{\chi}_{1}^{ \pm}$decay to leptons, and we can use almost all of the cascade events in our analysis.

\section{HIGGSINO-LIKE CASCADES}

In region $3, \tilde{\chi}_{2}^{0}$ and $\tilde{\chi}_{1}^{ \pm}$are again close in mass, but now, as suggested in Eq. 9, they are also close to the LSP in mass. We will take as our representative point $\left(\mu, M_{2}\right)=$ 
$(-100 \mathrm{GeV}, 700 \mathrm{GeV})$, where the masses of $\tilde{\chi}_{1}^{0}, \tilde{\chi}_{2}^{0}$, and $\tilde{\chi}_{1}^{ \pm}$are $98.3,110.9$, and $106.1 \mathrm{GeV}$, respectively. The branching ratios of left- and right-handed squarks to the LSP are now both $\sim 10 \%-20 \%$. The analysis for left- and right-handed squarks is similar, and we will consider only the left-handed below.

The quark jet energy distribution is shown in Fig. 10. The large low energy hump is composed of the soft quarks produced in the decays between the neutralinos and chargino. The primary vertex quarks have the flat energy distribution we expect, but this is really a superposition of decays to three different particles with slightly different masses. We see that the primary and secondary vertex quark jets are well-separated in energy, and we can isolate the primary quark jets with a simple jet energy cut at $30 \mathrm{GeV}$. In fact, we again have not included the decays of $\tilde{\chi}_{2}^{0}$ to $\tilde{\chi}_{1}^{ \pm}$in the Monte-Carlo simulation. This omission is physically unwarranted, as such decays no longer suffer the pronounced phase space suppression relative to the decays of $\tilde{\chi}_{2}^{0}$ and $\tilde{\chi}_{1}^{ \pm}$to the LSP. However, these decays will only increase the number of soft jets in the low energy hump, which will be eliminated with the energy cut anyway. We have also assumed $m_{\tilde{l}}=200 \mathrm{GeV}$ and $m_{h^{0}}=110 \mathrm{GeV}$. Other values will change the shape of the low energy hump, but again this is irrelevant after the energy cut.

We must now determine the squark mass from the jet energies. Unlike in the previous sections, we cannot use the $m_{\tilde{q}}^{\min }$ distribution, because for a given quark jet, we cannot tell if its primary vertex partner was $\tilde{\chi}_{2}^{0}$ or $\tilde{\chi}_{1}^{ \pm}$, and the $5 \mathrm{GeV}$ mass difference between these two is now significant compared to the the accuracy with which we hope to measure the squark masses. We will therefore simply use the jet energy distribution for our likelihood fit. Our strategy will then rely on the assumption that we know the neutralino and chargino parameters. The $\tilde{\chi}^{0}$ and $\tilde{\chi}^{ \pm}$masses will be quite accurately determined and will fix the relative positions of the endpoints of the energy distributions. Slepton and chargino studies should be able to determine the parameters $\mu$ and $M_{2}$, which will determine the branching ratios of the squarks. In the Higgsino-like region, it may be difficult to determine $M_{2}$ precisely, but since the branching ratios are relatively insensitive to $M_{2}$, we nevertheless also expect to determine the branching ratios accurately. Given these assumptions, we take the neutralino/chargino masses and branching ratios as inputs in our analysis. The only unknown is then the squark mass, and a $\ln \mathcal{L}$ fit gives us the squark mass to within $1.2 \mathrm{GeV}$ at $95 \%$ CL.

Region 3 is the only region where the corresponding $\mu>0$ region has different decay properties. In the $\mu>0$ region, all types of squarks decay to the LSP with branching ratios $\gtrsim 80 \%$. We can therefore use the direct decays in this region, and the analysis is actually simpler.

\section{HIGHER SQUARK MASSES}

So far we have been studying squarks with mass $\sim 220 \mathrm{GeV}$. Of course, squarks may be significantly more massive than this, and it is therefore important to consider the applicability of the preceding analysis to the case of higher mass squarks and higher energy colliders. With slight modifications, we will see that it is straightforward to adapt the analysis to higher energies. We note, however, that as the squark mass rises, our assumption of $m_{\tilde{q}}<M_{3}$ becomes more and more disfavored if one subscribes to the theoretical prejudice of a universal scalar mass. 
To proceed, we will consider the case of a $1 \mathrm{TeV}$ collider with luminosity $30 \mathrm{fb}^{-1} /$ year. Squarks with mass near the kinematic limit of $500 \mathrm{GeV}$ can be studied; we will take $m_{\tilde{q}_{L}}=$ $400 \mathrm{GeV}$ and $m_{\tilde{q}_{R}}=390 \mathrm{GeV}$. The $\left(\mu, M_{2}\right)$ plane may be divided into regions as before (see Fig. 11). All of the region boundaries move to higher $|\mu|$ and $M_{2}$, except the boundary between regions $2 \mathrm{a}$ and $2 \mathrm{~b}$. The boundary at which gluinos become less massive than squarks moves up to $M_{2} \approx 121 \mathrm{GeV}$. In addition, renormalization group equatons coupled with the desert hypothesis now imply $M_{2} \lesssim 200 \mathrm{GeV}$. Of course, region 4 , the region of complicated cascade decays, becomes large. A full treatment of this region would demand a significantly more complicated analysis.

The squark decays of regions $1,2 \mathrm{~b}$, and 3 are qualitatively similar to those in the lower energy case we considered earlier, and therefore similar analyses are applicable. An important difference for our analysis, however, is that region $2 \mathrm{a}$ is no longer a thin strip, and consequently the branching ratio to the LSP is small throughout most of region 2a. In the previous discussion, the branching ratio to the LSP, even for left-handed squarks, was substantial in region $2 \mathrm{a}$, and we based our analysis on the abundance of direct LSP decay. We see that this convenient feature does not persist to cases of higher squark masses and higher energy colliders, and for the case of $400 \mathrm{GeV}$ squarks we must use cascade decays for left-handed squarks in region $2 \mathrm{a}$.

To study this in detail, we again choose the point $\left(\mu, M_{2}\right)=(-500 \mathrm{GeV}, 200 \mathrm{GeV})$. Let us consider first the scenario when $m_{\tilde{l}}=380 \mathrm{GeV}$ and $m_{h^{0}}=110 \mathrm{GeV}$, so sleptons and Higgs bosons are too massive to be on-shell. In this case, the branching ratios are

$$
\begin{array}{ll}
\operatorname{BR}\left(\tilde{u}_{L} \rightarrow u \tilde{\chi}_{1}^{0}\right)=5 \%, & \operatorname{BR}\left(\tilde{d}_{L} \rightarrow d \tilde{\chi}_{1}^{0}\right)=2 \%, \\
\operatorname{BR}\left(\tilde{u}_{L} \rightarrow u \tilde{\chi}_{2}^{0}\right)=63 \%, & \operatorname{BR}\left(\tilde{d}_{L} \rightarrow d \tilde{\chi}_{2}^{0}\right)=66 \%, \\
\operatorname{BR}\left(\tilde{u}_{L} \rightarrow d \tilde{\chi}_{1}^{+}\right)=32 \%, & \operatorname{BR}\left(\tilde{d}_{L} \rightarrow u \tilde{\chi}_{1}^{-}\right)=32 \%,
\end{array}
$$

and

$$
\begin{array}{ll}
\operatorname{BR}\left(\tilde{\chi}_{2}^{0} \rightarrow q \bar{q} \tilde{\chi}_{1}^{0}\right)=68 \%, & \operatorname{BR}\left(\tilde{\chi}_{1}^{ \pm} \rightarrow q^{\prime} \bar{q} \tilde{\chi}_{1}^{0}\right)=66 \% \\
\operatorname{BR}\left(\tilde{\chi}_{2}^{0} \rightarrow l \bar{l} \tilde{\chi}_{1}^{0}\right)=11 \%, & \operatorname{BR}\left(\tilde{\chi}_{1}^{ \pm} \rightarrow l \nu \tilde{\chi}_{1}^{0}\right)=34 \% \\
\operatorname{BR}\left(\tilde{\chi}_{2}^{0} \rightarrow \nu \bar{\nu} \tilde{\chi}_{1}^{0}\right)=21 \% &
\end{array}
$$

The cascade decays dominate, and unfortunately, the on-shell $W$ and $Z$ diagrams result in a predominance of hadronic decay products, making it more difficult to identify the primary vertex jets. However, when there are only two quark jets, we know that these came from the primary vertex, and of the 2333 events that pass the $\not\rangle_{T}$ and $\theta_{\text {acop }}$ cuts, there are still 478 2 -jet events. Applying the same analysis to these events as was applied to the cascade events of region $2 \mathrm{~b}$ in Sec. $\mathrm{V}$, we find that the squark masses can be determined to $4.8 \mathrm{GeV}$ at 95\% CL. We therefore find that despite the predominance of cascades with hadronic decay products, we again expect to measure the squark masses to an accuracy of about $1 \%$.

It is tempting to try to improve our statistics by using some of the events with more than 2 jets. For these events, we can try to reconstruct the on-shell $W$ s and $Z$ s from jet pair invariant masses and thereby determine which quark jets are produced at the primary vertex. With six jets and the detector resolution of $50 \%$ assumed above, it is very difficult to determine with any certainty which two of the fifteen possible quark pairs have the correct invariant masses to be $W$ and $Z$ decay products. However, for the 996 four jet events, the determination is much easier. For these events, we accept only those events where exactly 
one of the six quark pairs has invariant mass within $10 \%$ of either $m_{W}$ or $m_{Z} .29 \%$ of the 4 jet events pass this invariant mass cut, and of these, fewer than $1 \%$ have misidentified $W$ s or $Z$ s. Therefore, in $29 \%$ of the $4 q$ and all of the $2 q$ events we can identify the primary quarks.

Of these 779 events, $67 \%$ are double cascade events. We then calculate $m_{\tilde{q}}^{\min }$ for each event and assume $m_{\tilde{\chi}}^{\text {primary }}=m_{\tilde{\chi}_{1}^{ \pm}}$. Demanding that this hypothesis be kinematically consistent leaves 647 events, of which $80 \%$ are double cascades. The $\ln \mathcal{L}$ fit to the $m_{\tilde{q}}^{\min }$ distribution gives the squark mass to $4.4 \mathrm{GeV}$ at $95 \% \mathrm{CL}$, a slight improvement of our previous result.

It is important to note that backgrounds to the 4-jet events are large and may make the 4-jet events difficult to isolate. In particular, if it is necessary to cut events with a pair of jets whose invariant mass is near the $W$ or $Z$ mass to reduce the background, the analysis of the preceding paragraph is of course not possible. In addition, if $h^{0}$ is light enough to be on-shell in the cascade diagrams, $b$ quarks will dominate the decay products and one cannot anti-tag bottom quarks. However, by using a combination of $b$-tagging and $h^{0}$ mass reconstruction one might hope to isolate the primary vertex quarks. On the other hand, the presence of light sleptons would make the analysis easier, since there are then more lepton decay products and more 2-jet events.

Measurements in region $2 \mathrm{~b}$ with high $m_{\tilde{l}}$ and high $m_{h^{0}}$ will be slightly degraded for the same reasons as in 2a, namely many cascades with few leptonic decays. One would again like to put the large fraction of events with more than two jets to use. Unfortunately, for these events, one will generally not be able to identify the primary quark jets, as there are no on-shell $W$ and $Z$ decays to reconstruct. However, it may still be possible to get information from these events. For example, noting that jets from cascade decays have a smooth energy distribution concentrated at lower energies, if one plots the distribution of 1 jet energies, one might be able to discern the endpoints of the primary decay jet distribution above the tail from the cascade jets. In the pessimistic high $m_{\tilde{l}}$ case we are considering, most events will include many jets, and a realistic study would require an accurate simulation of hadronization effects and jet reconstruction.

\section{ADDITIONAL REMARKS AND CONCLUSIONS}

In our whole analysis above, we have set $\tan \beta=2$. Raising $\tan \beta$ has little effect on the squark branching ratios in the $\mu>0$ part of the parameter plane, and its effect on branching ratios in $\mu<0$ regions is to make then more similar to $\mu>0$ regions. For example, when $\tan \beta=20$, the only large effect is that the branching ratio to the LSP in region 3 grows to $\approx 80 \%$. Thus, the decay patterns are nothing new, and by exploring all regions of the plane for $\tan \beta=2$, we have simultaneously roughly analyzed the case of higher $\tan \beta$.

In this paper we have begun to explore the prospects for measuring squark masses at future $e^{+} e^{-}$colliders, within the context of the MSSM. We have shown that, even if squarks choose complex decay patterns, these machines do offer opportunities for making squark mass measurements at the level of a few GeV. Such precision measurements would be invaluable for probing the mechanism of supersymmetry breaking in deeper underlying theories. Interesting questions for future research include how more realistic physics simulations would affect this picture and how squark masses would be studied in the case that gluinos are the dominant decay channel. 


\section{ACKNOWLEDGMENTS}

It is a pleasure to thank M. Peskin for suggesting this topic, and we are grateful to him and to H. E. Haber and T. Barklow for many helpful discussions. 


\section{REFERENCES}

[1] For reviews of SUSY and the MSSM, see H.P. Nilles, Phys. Rep. 110, 1 (1984); P. Nath, R. Arnowitt, and A.H. Chamseddine, Applied $N=1$ Supergravity, ICTP Series in Theoretical Physics, Vol. 1 (World Scientific, Singapore, 1984); H.E. Haber and G.L. Kane, Phys. Rep. 117, 75 (1985); X. Tata, in The Standard Model and Beyond, proceedings of the Ninth Symposium on Theoretical Physics, Mt. Sorak, Korea, 1990, edited by J.E. Kim, (World Scientific, River Edge, New Jersey, 1991), p. 304.

[2] M.B. Einhorn and D.R.T. Jones, Nucl. Phys. B196, 475 (1982); U. Amaldi et al., Phys. Rev. D 36, 1385 (1987); U. Amaldi, W. de Boer, and H. Furstenau, Phys. Lett. B260, 447 (1991); P. Langacker and M. Luo, Phys. Rev. D 44, 817 (1991); J. Ellis, S. Kelley, and D.V. Nanopoulos, Phys. Lett. 287B, 95 (1992); ibid., Nucl. Phys. B373, 55 (1992); F. Anselmo, L. Cifarelli, A. Peterman, and A. Zichichi, Nuovo Cim. 104A, 1817 (1992); ibid., 105A, 581 (1992); ibid., 105A, 1201 (1992).

[3] JLC Group, JLC-I, Tsukuba, Japan, 1992 (KEK Report 92-16, Tsukuba, 1992).

[4] S. Orito, talk delivered at the Workshop on Physics and Experiments with Linear $e^{+} e^{-}$ Colliders, Waikoloa, Hawaii, 1993 (proceedings to be published).

[5] T. Kon, ibid.

[6] T. Tsukamoto, ibid.

[7] C. van der Velde, ibid.

[8] CDF Collaboration, F. Abe et al., Phys. Rev. Lett. 69, 3439 (1992).

[9] C. Ahn et al., SLAC Report No. 329 (1988).

[10] $e^{+} e^{-}$Collisions at $500 \mathrm{GeV}$ : The Physics Potential, Proceedings of the Workshop Munich, Annecy, Hamburg, 1991, edited by P. Zerwas, DESY Report No. 92-123 (1992).

[11] H. Baer, J. Ellis, G. Gelmini, D.V. Nanopoulos, and X. Tata, Phys. Lett. 161B, 175 (1985).

[12] G. Gamberini, Z. Phys. C30, 605 (1986).

[13] H. Baer, M. Drees, D. Karatas, and X. Tata, in Proceedings of the 1987 Berkeley Workshop on Experiments, Detectors and Experimental Areas for the Supercollider, edited by R. Donaldson and M. Gilchriese (World Scientific, Singapore, 1988), p. 210.

[14] H. Baer, X. Tata, and J. Woodside, Phys. Rev. Lett. 63, 352 (1989); ibid., Phys. Rev. D 44, 207 (1991).

[15] A. Bartl, W. Majerotto, B. Mösslacher, and N. Oshimo, Z. Phys. C52, 477 (1991).

[16] A. Bartl, W. Majerotto, B. Mösslacher, N. Oshimo, and S. Stippel, Phys. Rev. D 43, 2214 (1991).

[17] J.F. Gunion and H.E. Haber, Nucl. Phys. B272, 1 (1986); errata: SCIPP-92/59, UCD 92-31 (1992).

[18] L. Girardello and M.T. Grisaru, Nucl. Phys. B194, 65 (1982).

[19] J. Ellis and D.V. Nanopoulos, Phys. Lett. 110B, 44 (1982); T. Inami and C.S. Lim, Nucl. Phys. B207, 533 (1982); M.J. Duncan and J. Trampetic, Phys. Lett. 134B, 439 (1984).

[20] M. Dine, A. Kagan, and R. Leigh, SLAC-PUB-6147, SCIPP-93/04 (1993).

[21] Y. Nir and N. Seiberg, RU-93-16, WIS-93/37 (1993).

[22] S.P. Ahlen et al., Phys. Lett. 195B, 603 (1987); D. Caldwell et al., Phys. Rev. Lett. 61, 510 (1988); H. Baer, M. Drees, and X. Tata, Phys. Rev. D 41, 3414 (1990).

[23] K. Inoue, A. Kakuto, H. Komatsu and S. Takeshita, Prog. Theor. Phys. 68, 927 (1982). 
[24] J.-F. Grivaz, in Proceedings of the Workshop on Physics and Experiments with Linear Colliders, Saariselka, Finland, 1991, Vol. 1, edited by R. Orava, P. Eerola, and M. Nordberg (World Scientific, River Edge, New Jersey, 1992), p. 353.

[25] Particle Data Group, K. Hikasa et al., Phys. Rev. D 45 S1 (1992).

[26] ALEPH Collaboration, D. Décamp et al., Phys. Rep. 216, 253 (1992).

[27] J.F. Gunion and H.E. Haber, Phys. Rev. D 37, 2515 (1988). 


\section{FIGURES}

FIG. 1. The number of squark pairs of the first two generations produced at a $500 \mathrm{GeV} e^{+} e^{-}$ collider with polarized beams and an integrated luminosity of $10 \mathrm{fb}^{-1}$ for each beam polarization. The four helicity combinations plotted are $e_{L, R}^{-} e^{+} \rightarrow \tilde{q}_{L, R} \overline{\tilde{q}}$.

FIG. 2. The $\left(\mu, M_{2}\right)$ plane divided into regions with similar squark decay channels, given $\tan \beta=2$ and $m_{\tilde{q}}=220 \mathrm{GeV}$. The squark decays of the various regions are described in the text. The points of parameter space that we consider in detail in the text are marked. The condition $m_{\tilde{q}}<M_{3}$ is true only above the dotted line at $M_{2}=67 \mathrm{GeV}$.

FIG. 3. The jet energy distribution resulting from squark decays in region 1 at the point $\left(\mu, M_{2}\right)=(-500 \mathrm{GeV}, 300 \mathrm{GeV})$. Each jet produced in such events is binned individually. Cuts and detector resolution effects alter the flat shape and make determination of the endpoints more difficult. The solid (dashed) histogram represents events with $e_{L}^{-}\left(e_{R}^{-}\right)$polarized beams. The integrated luminosity assumed is $5 \mathrm{fb}^{-1}$ per polarization, and the bin size is $4 \mathrm{GeV}$.

FIG. 4. Determination of minimum kinematically-allowed squark mass from the two visible quark momenta and the LSP mass. The momenta label the particles of $\tilde{q}\left(p_{1}\right) \rightarrow \tilde{\chi}_{1}^{0}\left(p_{3}\right) q\left(p_{4}\right)$ and $\tilde{q}\left(p_{2}\right) \rightarrow \tilde{\chi}_{1}^{0}\left(p_{5}\right) q\left(p_{6}\right)$. The momenta of the two undetected LSPs are constrained to lie on the circle $C$.

FIG. 5. The distribution of $m_{\tilde{q}}^{\min }$, the minimum allowed squark mass for a given event, in region 1 at the point $\left(\mu, M_{2}\right)=(-500 \mathrm{GeV}, 300 \mathrm{GeV})$. The distribution for $e_{L}^{-}\left(e_{R}^{-}\right)$polarized beams is given by the solid (dashed) histogram and is sharply peaked at the actual $\tilde{q}_{L}\left(\tilde{q}_{R}\right)$ mass of 220 (210) $\mathrm{GeV}$. The integrated luminosity assumed is $5 \mathrm{fb}^{-1}$ per polarization, and the bin size is $5 \mathrm{GeV}$.

FIG. 6. Contours of the branching ratios a) $\operatorname{BR}\left(\tilde{u}_{L} \rightarrow u \tilde{\chi}_{1}^{0}\right)$ and b) $\operatorname{BR}\left(\tilde{u}_{R} \rightarrow u \tilde{\chi}_{1}^{0}\right)$ in percent in the $\left(\mu, M_{2}\right)$ plane. In the gaugino-like region $2, \tilde{u}_{L}$ decays predominantly via cascades, while $\tilde{u}_{R}$ is seen to decay primarily to $\tilde{\chi}_{1}^{0}$ even though other on-shell decays are kinematically allowed.

FIG. 7. Jet energy distributions at the point $\left(\mu, M_{2}\right)=(-500 \mathrm{GeV}, 200 \mathrm{GeV})$ in region 2 a. Every jet produced in the Monte-Carlo squark decay events is binned individually. The three components are a large peak of soft quarks emanating from squarks decaying to $\tilde{\chi}_{2}^{0}$ and $\tilde{\chi}_{1}^{ \pm}$(long dashes), a flat distribution from direct LSP decays (solid), and a wide hump from hadronic $W$ and $Z$ decays (short dashes). The total distribution is given by the dotted histogram. The analysis is independent of the soft jets. The integrated luminosity assumed is $5 \mathrm{fb}^{-1}$ per polarization, and the bin size is $4 \mathrm{GeV}$. 
FIG. 8. The total jet energy distribution at the point $\left(\mu, M_{2}\right)=(-500 \mathrm{GeV}, 200 \mathrm{GeV})$ (solid), and the bottom quark energy distribution resulting from the top quark pair production background (dashes). In the bottom quark spectrum, we have removed all $b$ quarks produced in top events in which at least one $b$ quark has been successfully tagged. We assume $m_{\text {top }}=150 \mathrm{GeV}$ and a $b$-tagging efficiency of $80 \%$ [3].

FIG. 9. The Feynman diagrams for three-body $\tilde{\chi}_{2}^{0}$ and $\tilde{\chi}_{1}^{ \pm}$decays mediated by $h^{0}$, charged sleptons and sneutrinos, squarks, and $Z$ and $W$ bosons.

FIG. 10. Jet energy distributions at the point $\left(\mu, M_{2}\right)=(-100 \mathrm{GeV}, 700 \mathrm{GeV})$ in region 3 . The solid (dashed) histogram represents events with $e_{L}^{-}\left(e_{R}^{-}\right)$polarized beams. The soft hump of secondary vertex jets is well-separated from the primary vertex jet distribution. The integrated luminosity assumed is $5 \mathrm{fb}^{-1}$ per polarization, and the bin size is $4 \mathrm{GeV}$.

FIG. 11. The $\left(\mu, M_{2}\right)$ plane divided into regions with similar squark decay channels, given $\tan \beta=2$ and $m_{\tilde{q}}=400 \mathrm{GeV}$. The squark decays of the various regions are as in Fig. 2 and are given in the text. The point in region $2 \mathrm{a}$ that we consider in detail is marked. The condition $m_{\tilde{q}}<M_{3}$ is true only above the dotted line at $M_{2}=121 \mathrm{GeV}$. 
This figure "fig1-1.png" is available in "png" format from: http://arxiv.org/ps/hep-ph/9310211v1 
This figure "fig2-1.png" is available in "png" format from: http://arxiv.org/ps/hep-ph/9310211v1 
This figure "fig1-2.png" is available in "png" format from: http://arxiv.org/ps/hep-ph/9310211v1 
This figure "fig2-2.png" is available in "png" format from: http://arxiv.org/ps/hep-ph/9310211v1 
This figure "fig1-3.png" is available in "png" format from: http://arxiv.org/ps/hep-ph/9310211v1 
This figure "fig2-3.png" is available in "png" format from: http://arxiv.org/ps/hep-ph/9310211v1 
This figure "fig1-4.png" is available in "png" format from: http://arxiv.org/ps/hep-ph/9310211v1 
This figure "fig2-4.png" is available in "png" format from: http://arxiv.org/ps/hep-ph/9310211v1 
This figure "fig1-5.png" is available in "png" format from: http://arxiv.org/ps/hep-ph/9310211v1 
This figure "fig2-5.png" is available in "png" format from: http://arxiv.org/ps/hep-ph/9310211v1 
This figure "fig1-6.png" is available in "png" format from: http://arxiv.org/ps/hep-ph/9310211v1 